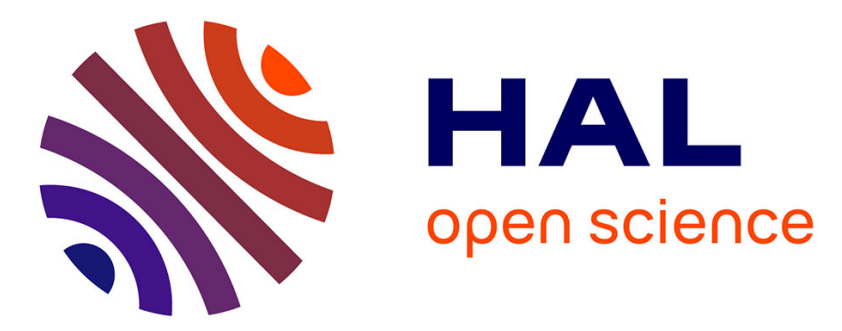

\title{
Optimal sizing of an electrical machine using a magnetic circuit model: application to a hybrid electrical vehicle
}

Vincent Reinbold, Emmanuel Vinot, Lauric Garbuio, Laurent Gerbaud

\section{To cite this version:}

Vincent Reinbold, Emmanuel Vinot, Lauric Garbuio, Laurent Gerbaud. Optimal sizing of an electrical machine using a magnetic circuit model: application to a hybrid electrical vehicle. IET Electrical Systems in Transportation, 2016, 6 (1), pp. 27-33. 10.1049/iet-est.2015.0008 . hal-01301720

\author{
HAL Id: hal-01301720 \\ https://hal.science/hal-01301720
}

Submitted on 4 Feb 2019

HAL is a multi-disciplinary open access archive for the deposit and dissemination of scientific research documents, whether they are published or not. The documents may come from teaching and research institutions in France or abroad, or from public or private research centers.
L'archive ouverte pluridisciplinaire HAL, est destinée au dépôt et à la diffusion de documents scientifiques de niveau recherche, publiés ou non, émanant des établissements d'enseignement et de recherche français ou étrangers, des laboratoires publics ou privés. 


\title{
Optimal sizing of an electrical machine using a magnetic circuit model :
}

\author{
application to a hybrid electrical vehicle
}

\author{
Reinbold Vincent*, Vinot Emmanuel ${ }^{\dagger}$, Garbuio Lauric* and Gerbaud Laurent* \\ * Grenoble University, G2eLab (CNRS UMR5269, INPG, UJF), \\ F-38142 Cedex, Grenoble, France, vincent.reinbold@g2elab.grenoble-inp.fr \\ † IFSTTAR, 25 Av. François Mitterrand, 69675 Bron - France, \\ emmanuel.vinot@ifsttar.fr
}

February 21, 2017

\begin{abstract}
Numerous researches about hybrid electrical vehicles (HEV) deal with topologies, technologies, sizing and control. These aspects allow reducing transportation costs and environmental impacts. The paper focuses on the sizing of the electrical machine of the HEV, taking into account its surroundings: the hybrid system, the driving cycle and an optimal energy management. In the paper, the parallel hybrid electrical vehicle is the study case. In a classical HEV design process, a scaling factor is usually applied on an efficiency map model to fix the standard power of the electrical machine. The efficiency and the maximum torque power are scaled using a linear dependency on the rated maximum power. But this method has some disadvantages. The paper proposes two formulations of a scaling model based on a magnetic circuit model (MCM) with one or 10 parameters. Then, the MCM is involved in a multi-objective optimization process of the HEV. This process is a global sizing process using dynamic programming as an optimal energy management. Optimal sizings of the hybrid vehicle are then proposed for various driving conditions.
\end{abstract}

\section{Introduction}

The fuel consumption of a hybrid electric vehicle (HEV) depends on: the driving cycle (i.e. the use of the vehicle), the energy management and the power-train components, e.g. the 
internal combustion engine (ICE), the electrical machine (EM) and gear ratios. In HEVs, there is a strong interaction between the driving cycle, the energy management and components. In particular, the components efficiency and performances highly interfere with the energy management which in return fixes the operation of the components. Previous studies on optimizing the drive train component of HEVs usually use predefined energy management strategies, such as rule-based strategies [1]. Thus, during the optimization process, the different vehicle sizings will benefit differently from the strategy. Therefore, to optimize the electrical machine (EM), the whole hybrid system must be taken into account and a joint optimization of the system component and the energy management must be performed. This approach was developed in previous studies for energetic parameters such as standard power and ratios [2-9]. One limitation of this approach is that it usually uses efficiency maps. The paper aims to take into account the geometrical parameters of the EM in order to adapt the design of the EM to the use of the HEV.

In order to estimate electrical machine performances, finite element methods (FEM) are generally used by designers to predict core losses and efficiency for various operating points and geometries. However, their estimation is generally time-consuming, which makes the use of the FEM prohibitive in an iterative optimization process of the global system. Thus, a map scaling factor model (MSFM) based on the knowledge of an efficiency map is generally used $[5,6]$. This way, the standard power of the EM can vary easily in the sizing process. However, depending on whether the optimization goes far or close to the EM initial power, the results can be accurate or not. In addition, there is no direct link between the standard power of the EM and the geometrical proprieties of the machine. Thus, the paper proposes a compromise between the FEM and the MSFM introducing a magnetic circuit model (MCM). A comparison between the MSFM and the MCM was done in a previous study in an optimization process. As a main result, the MSFM is not adapted to a sizing process of the EM [10].

This paper presents the development of a magnetic circuit model and its use in an optimal sizing process. Two models are proposed and compared, using only one scaling factor or ten uncorrelated geometrical parameters.

Firstly, a general presentation of the parallel hybrid electric vehicle is made in section 2. Secondly, the two sizing methods (MSFM and MCM) are described in section 3. Afterwards, the optimal energy management problem is presented in section 4 . Then, the sizing problem of the parallel-HEV is defined in section 5. Lastly, optimal sizing results 
are presented for three driving conditions, and a full operating analysis of the optimal EM is proposed for an urban driving cycle.

\section{Parallel Hybrid Electric Vehicle}

The parallel hybrid electric vehicle is a well-known HEV topology which involves an electrical machine (EM) coupled to an internal combustion engine (ICE) by a coupling device (see Fig. 1). Both the ICE and the EM deliver power to the drive train. The energy flow is parallel.

Such an architecture can operate in different mode:

- an all electric mode (clutch1 closed and clutch2 open) which is used to propel the vehicle at low power or during electrical regenerative braking.

- In hybrid mode clutch 1 and 2 are closed. In this mode the EM can be used to help the ICE in case of high power requirement. It can also be used as a generator to charge the battery. This can allow the ICE to operate on better specific full consumption and the stored energy can be used later.

It is clear that the system needs to be managed to fix the operating mode. In fact, the energy management is a key point of the efficiency of hybrid vehicle $[1,11,12]$.

Energy management can be performed using a rule based heuristic method [7-9], but this can have a non-monotonic effect depending of the size of the components $[3,6]$. The management law can be efficient for one sizing and not for another. To avoid this, in the scope of this paper, the author choses to use the optimal management method giving the fuel consumption on a driving cycle (see section 4).

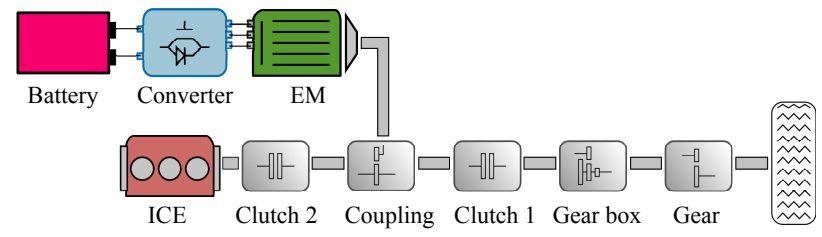

Figure 1: Parallel HEV architecture

The paper aims to optimize a parallel-HEV. The initial sizing of which is based on a real small passenger car (see Tab. 8) coupled with a known electric machine. The basic requirements for an electrical machine for HEV are : power density, efficiency on various operating points, controllability, reliability, technological maturity and cost $[13,14]$. There 


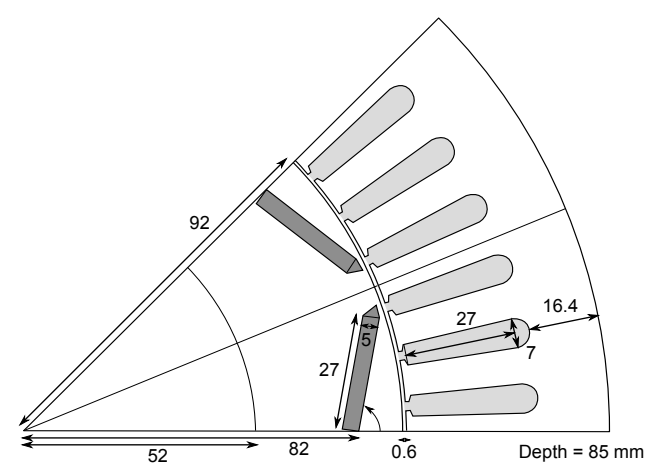

Figure 2: Geometrical representation of one eighth of the initial IPMSM

is no global consensus about which types of electrical machine is to be used for parallelHEV. Nevertheless, the permanent motor (PM) present a higher power density and a higher maximal efficiency than other type of EM, like the induction machine, the DC machine or the switch reluctance machine. That is why we choose to study an interior permanent magnet synchronous motor (IPMSM) such as those usually used in hybrid or electric vehicles (Toyota hybrid system for instance). It is noted that the proposed method can be applied to other type of EM if a MCM is developed.

The initial characteristics of the EM are shown in Fig. 2. The initial geometrical characteristics and manufacturing assessment come from pictures and measurements of the Toyota Prius 2004 electrical machine [15-17].

The following section deals with a sizing method of the EM.

\section{Scaling models of the electrical machine}

In a vehicle simulation context, the efficiency map is a useful data, usually obtained from measurements or from a fine model (like FEM). In a sizing context, it is commonly used to simulate instantaneous fuel rate for ICE and the efficiency of the EM with respect to the speed and the torque. The size of the EM varies according to a scaling factor applied to the torque and losses.

There is no direct link between the nominal power $P_{b, k}$ and the geometrical parameters. Nevertheless, in order to compute the new geometrical characteristics of the scaled machine, it is usually assumed that the power density is constant and lengths proportional to $k^{1 / 3}[5,18]$. Mass moments of inertia have units of dimension $M \cdot L^{2}$, this leads to a moment of inertia proportional to $k^{5 / 3}$. 
The scaling factor model based on an efficiency map can be a very accurate model close to the initial EM power. But this model, usually obtained from measurements, does not give information about the geometrical characteristics of the EM. Furthermore, the accuracy of this model is decreasing as the factor is far from 1. In parallel HEVs, the standard power of the EM is not constrained directly by the dynamic performances of the vehicle. Thus, its standard power can vary from half to twice the initial standard power. In addition, this lack of accuracy can have a negative impact on the global sizing of the vehicle. A wrong assessment of the weight and the efficiency map can lead to a sub-optimal sizing of the vehicle [10].

The relation between the standard power and the geometrical characteristics of the EM is not trivial. A scaling factor applied to an efficiency map appears to be questionable in some cases. Thus, the paper introduces a parametrized magnetic circuit model of the EM, which will be used in the global design of the system.

\subsection{Magnetic circuit model}

\subsubsection{Modelling}

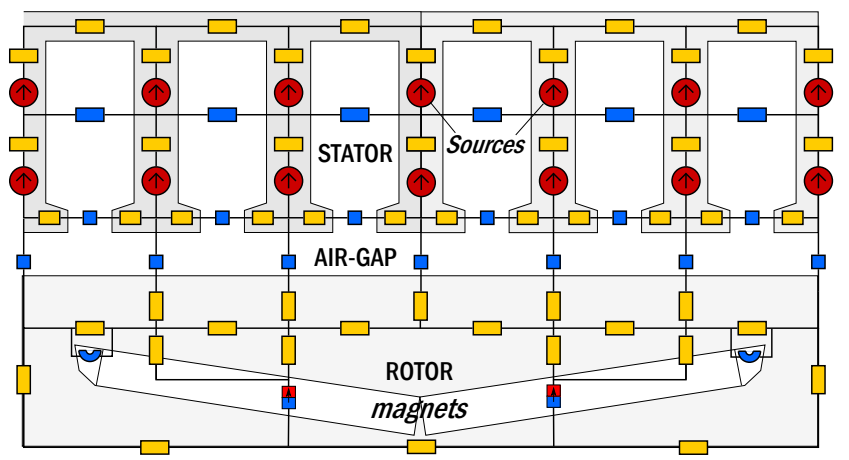

Figure 3: Magnetic circuit model of one eighth of the IPMSM

The magnetic circuit model uses a magneto-electrical equivalence. It is based on the knowledge of the geometrical parameter set named $\mathbb{X}$, e.g. air-gap length, magnet sizes, stator external radius and winding characteristics (see Fig. 2). This leads to a magnetic circuit (see Fig. 3) where each reluctance depends on the geometrical parameters (a reluctance is the magnetic equivalence to an electric resistance) [19]. Magnetic sources depend on the peak current $I_{e m}$ and the internal electrical angle $\delta$ i.e. the oriented angle between the current and the quadratic axes. A Newton-Raphson method is used to solve 
the circuit problem [19], and gives the direct and quadratic flux linkage $\phi_{d}, \phi_{q}$ :

$$
\left\{\begin{array}{l}
\phi_{d}=\phi_{d}\left(\mathbb{X}, I_{e m}, \delta\right) \\
\phi_{q}=\phi_{q}\left(\mathbb{X}, I_{e m}, \delta\right)
\end{array}\right.
$$

In the Park's reference frame (a rotating bidirectional reference frame), the direct and the quadratic voltage $v_{d}, v_{q}$ are then deduced:

$$
\left\{\begin{array}{l}
v_{d}=R_{s} i_{q}-\omega \phi_{q} \\
v_{q}=R_{s} i_{d}+\omega \phi_{d}
\end{array}\right.
$$

where $\omega=p \Omega$ is the electrical pulsation and $p$ is the number of pole pairs. The calculation of the electrical power $P_{e}$ leads to:

$$
P_{e}=\frac{3}{2} \Re\left[I_{\text {em }} V^{*}\right]=P+\underbrace{P_{J}+P_{\text {iron }}+P_{f}}_{\text {losses }}
$$

where $P_{J}, P_{\text {iron }}$ and $P_{f}$, are respectively, the Joule, the iron and the mechanical losses. Note that the eddy current losses in the permanent magnets are neglected in this model since this machine has a theoretical null harmonic distortion that limits eddy current losses [20]. Furthermore, in a complex reference, the vector $I_{m e}$ and $V^{*}$ can be expressed as follows:

$$
\left\{\begin{array}{l}
I_{e m}=i_{d}+j i_{q} \\
V^{*}=v_{d}-j v_{q}
\end{array}\right.
$$

thus,

$$
\begin{aligned}
\Gamma_{e m} & =\frac{P}{\Omega}=\frac{P_{e}-P_{J}-P_{\text {iron }}-P_{f}}{\Omega} \\
& =\frac{3}{2} p\left(\phi_{d} i_{q}-\phi_{q} i_{d}\right)-\frac{\left(P_{\text {iron }}+P_{f}\right)}{\Omega_{e m}}
\end{aligned}
$$

In this study, the iron losses per volume in each reluctance $\left(p_{i}\right)$ are calculated thanks to a first harmonic Bertotti's model [21,22]:

$$
p_{i}=k_{h} f B_{i}^{2}+\sigma \frac{d^{2} \pi^{2}}{6} B_{i}^{2} f^{2}+8.67 k_{e} f^{1.5} B_{i}^{1.5}
$$

where $B_{i}$ is the maximum magnetic field in the reluctances, $f$ is the fundamental frequency, $d$ is the lamination thickness and $\sigma$ is the conductivity of the iron material. $k_{h}, k_{e}$ are 
respectively the hysteresis and the excess material constants. The mechanical losses are assumed to be proportional to the square of the external diameter of the machine, and therefore, can be expressed by the empirical expression [17]:

$$
P_{f}=\Omega\left(\frac{D_{e x t}}{0.2}\right)^{2}
$$

The optimal internal electrical angle, is calculated in order to reach the maximum torque per amps with respect to the voltage constraint.

A scaling model of the static converter has been also developed. These are computed thanks to an analytical model taking into account conduction and switching losses in diodes and IGBT [23].

Torque limitations are calculated by voltage and current constraints. For thermal issue, the maximum RMS current density allowed is $j_{\max }=12 \mathrm{~A} . \mathrm{mm}^{-1}$.

\subsubsection{Model validation}

A validation of the magnetic circuit model has been made using a Finite Element Method (FEM) with FLUX2D for an IPMSM machine with the geometry presented in Fig. 2. This fine model allows an accurate computation of torque and losses for different operating points. Since MCM is a first-harmonic model, a table comparing first harmonic voltage amplitude is proposed in Tab. 1. One can see the good agreement of the voltage for different speeds and current. Note that the internal angle of the current is fixed, thus, the voltage can be high for a speed higher than the base speed.

\begin{tabular}{c|c|cccc}
$\begin{array}{c}\text { Speed } \\
(\text { rpm })\end{array}$ & Model & \multicolumn{5}{|c}{ First harmonic voltage amplitude (V) } \\
\hline 600 & & $I_{e m}=0 A$ & $50 A$ & $100 A$ & $200 A$ \\
\hline & MCM & 102.0 & 112.4 & 117.8 & 139.1 \\
& FEM & 103.4 & 117.1 & 126.0 & 147.7 \\
\hline \multirow{2}{*}{200} & MCM & 204.0 & 226.0 & 228.3 & 266.2 \\
& FEM & 207.5 & 229.8 & 243.8 & 269.9 \\
\hline \multirow{2}{*}{400} & MCM & 408.0 & 438.0 & 449.3 & 520.9 \\
& FEM & 416.0 & 455.2 & 479.3 & 526.4
\end{tabular}

Table 1: First harmonic of the voltage comparison between MCM and FEM models

In order to validate the model of losses, a comparison of the statoric iron losses is shown in Tab. 2 for different operating points. The model used in the FEM model is a multi-harmonic Bertotti's model [24], whereas the MCM model uses a first-harmonic 
model [21]. One can see that trends are preserved and that relative error is kept under $20 \%$, which is reasonable for this simple model.

\begin{tabular}{c|c|ccc} 
Speed & Model & \multicolumn{4}{|c}{ Statoric iron losses (W) } \\
$\left(\right.$ tr.min $\left.^{-1}\right)$ & & $50 A$ & $100 A$ & $200 A$ \\
\hline 600 & MCM & 37.4 & 40.7 & 50.6 \\
& FEM & 38.1 & 43.8 & 53.3 \\
\hline 1200 & MCM & 84.4 & 91.6 & 113.9 \\
& FEM & 89.5 & 102.6 & 130.1 \\
\hline 2400 & MCM & 203.3 & 220.6 & 274.1 \\
& FEM & 230.0 & 261.5 & 342.8
\end{tabular}

Table 2: Iron losses comparison between MCM and FEM models

\subsection{Comparison between the MSFM and the MCM}

As presented in 3.1, the geometrical parameters $\mathbb{X}$ are not correlated. In an energetic sizing process or pre-sizing process, only homothetic transformations are usually considered. An alternative to the map scaling factor method is then introduced using a scaling factor on the geometrical parameters of the magnetic circuit model $\mathbb{X}$. A scaling MCM method is useful to perform a fair comparison with the MSFM [10]. It also can be compared with a more complicated method using uncorrelated parameters (see section 6).

The differences between the classical map scaling factor method and the proposed magnetic circuit scaling factor method in an optimization process are emphasized in [10]. In particular, note that the lack of accuracy of the MSFM introduces differences in the optimal sizing of the EM. Afterwards, the paper only focuses on the MCM. The results for the best operating points and standard operating points are presented in Tab. 3 .

\begin{tabular}{l|ccc|ccc} 
& \multicolumn{3}{|c|}{ MSFM } & \multicolumn{3}{c}{ MCM } \\
$k$ & $\eta_{b}(\%)^{a}$ & $\eta^{*}(\%)^{b}$ & $P_{b}(k W)$ & $\eta_{b}(\%)$ & $\eta^{*}(\%)$ & $P_{b}(k W)$ \\
\hline 1 & 89.7 & 94.9 & 58.8 & 89.7 & 94.9 & 58.8 \\
0,4 & 89.7 & 94.9 & 23.5 & 89.6 & 93.0 & 25.7 \\
1,3 & 89.7 & 94.9 & 76.4 & 92.5 & 95.3 & 73.1 \\
& \multicolumn{1}{c}{ efficiency of the standard operating point } \\
& ${ }^{b}$ efficiency of the best operating point
\end{tabular}

Table 3: Standard and maximum efficiency for the map scaling factor method (MSFM) and the magnetic circuit method (MCM) with, $k \in\{1,0.4,1.3\}$

The scaling factor on the geometrical parameters and on the current does not preserve the same magnetic operating as the initial EM. This phenomenon is mainly due to the non-linearity of the first magnetization curve of the magnetic material. As a result, one can see the negative evolution of the efficiency in the case $k<1$. 


\section{Optimal energy management}

To exclude the influence of the control strategy on component sizing, an optimal control method is used. It is an objective way to compare different sizings of hybrid vehicles $[5,25,26]$. The driving cycle is fixed and a priori known. In this configuration, the optimal control aims to determine:

- the battery current $I_{b a t}(t)$, which determines the power shared between the EM and the ICE;

- the clutch control $u_{c}(t)$, which determines the operating mode (hybrid or electrical);

- and the gear shifting control $k_{g b}(t)$.

The fuel consumption objective $J$ over the driving cycle can be expressed as follows:

$$
J=\int_{t_{0}}^{t_{f}} d_{J}\left(I_{b a t}(t), k_{g b}(t), u_{c}(t)\right) \mathrm{d} t
$$

where $d_{J}$ is the instantaneous fuel rate, and $t_{0}, t_{f}$ are respectively, the initial and the final time of the driving cycle.

For a charge sustaining vehicle (non rechargeable vehicle), the variation of the state of charge $(s o c)$ over the entire cycle is constraint to be equal to zero. Thus, an equality constraint is added to this problem:

$$
\Delta_{S O C}=\int_{t_{0}}^{t_{f}} d_{S O C}\left(I_{b a t}(t)\right) \mathrm{d} t \hat{=} 0
$$

In the paper, a dynamic programming algorithm is used [27]. This allows to solve the optimal power flow problem $\left(I_{b a t}(t)\right)$ and the discrete control problem $\left(k_{g b}(t)\right.$ and $\left.u_{c}(t)\right)$ while respecting the equality constraint (9). This method is based on the knowledge of the whole system: the driving cycle, the sizing of the vehicle, the component inertia, the efficiency map of the EM and instantaneous fuel rate map of the ICE. A similar presentation of the dynamic programming method is presented in $[5,26]$.

The following section deals with the dynamic programming method as an optimal energy management, in a sizing context. 


\section{$5 \quad$ Sizing problem}

The global problem can be seen as an optimization problem involving the sizing parameters and the optimal energy management. Indeed, the optimal trajectory $I_{b a t}(t)$ changes at each step of the sizing problem solving. A possible method to deal with this kind of problem is to determine the optimal control (see section 5.3) at each step of the sizing optimization.

\subsection{Optimization variables}

This study focuses on the electrical machine. However, as said in section 1, we have to take into account the other power train components that have impacts on the operating points of the EM, on the dynamic performances and on the fuel consumption. The optimization parameters taken into account in this study are : $N_{b a t}, P_{i c e}, P_{m e}, k_{c p l}$ and $k_{r e d}$ (see Tab. 8 for definitions). This study proposes to compare two sizing problem formulations described in Tab. 4 in terms of computation time, complexity and efficiency.

\begin{tabular}{l|cc} 
& $\mathbf{S P 1}$ & $\mathbf{S P 2}$ \\
\hline unknown & $N_{b a t}, P_{i c e}, k, k_{c p l}, k_{\text {red }}$ & $N_{b a t}, \mathbb{X}, V_{d c}, P_{i c e}, k_{c p l}, k_{\text {red }}$ \\
variables & $\mathbb{X}=k^{1 / 4} \mathbb{X}_{1}$ & - \\
subject to & $V_{\max }=\sqrt{k} \cdot V_{\max , 1}$ & -
\end{tabular}

Table 4: Sizing problem formulations

For the first formulation, noted SP1, the geometrical parameters of the electrical machine and the maximal voltage are related by a scaling factor $k . \mathbb{X}_{1}$ and $V_{\max , 1}$ are respectively the initial parameter set and the initial maximal voltage of the EM (see Fig. 2 and Tab. 8). This formulation leads to a unique shape of the electrical machine and only 5 unknown sizing variables, whereas the formulation SP2 considers the geometrical variables of the EM to be uncorrelated. Thus, the second formulation defines 15 unknown variables.

Using this two methods allows a fair comparison in terms of methodology and results (e.g. time to compute, fuel consumption, volume of the EM, raw materials, etc. see section $6)$.

\subsection{Constraints and objective functions}

In order to maintain constant comfort and security for all vehicle sizings, performance constraints have been added to the sizing problem: 
- the acceleration time from 0 to $100 \mathrm{~km} \cdot \mathrm{h}^{-1}, t_{0-100}<12 \mathrm{~s}$;

- the maximum speed of the vehicle on a flat road, $V_{\max }<140 \mathrm{~km} \cdot \mathrm{h}^{-1}$;

- the passing time of a truck driving at $80 \mathrm{~km} \cdot \mathrm{h}^{-1}, t_{d e p}<9 s$.

These constraints are computed with a forward approach [28]. For the SP2, some geometrical constraints are added in order to only have positive reluctances in the MCM.

The sizing of a HEV is usually a compromise between the cost-in-use and the raw material. To solve this problem, two objectives are introduced for the sizing problem :

- the fuel consumption (see eq. (8)),

- the battery module number in series $N_{b a t}$.

\subsection{Optimization strategy}

The sizing optimization is carried out by the Non-dominated Sorting Genetic Algorithm II (NSGA-II) introduced by K. Deb in [29]. This method, based on an evolutionary theory is used to perform conflicted multi-objective optimization. This algorithm leads to a nondominated set of solutions, called the Pareto front. A solution is called non-dominated, if none of the objective functions can be improved without degrading other objectives. In our case, we obtain a non-dominated set of sizing depending on the battery module number and the fuel consumption.

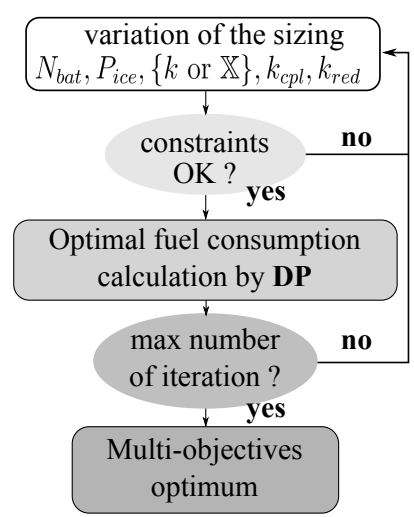

Figure 4: Optimization strategy - energy management as a sub-optimization problem

The optimization strategy is represented in Fig. 4 for both formulations. Note that the constraints are computed in a first place, then the optimal energy management is computed for each vehicle using dynamic programming. The optimization process stops when the maximum number of iterations is reached [29]. In our case, the convergence of the 
algorithm is acceptable after about $3.10^{4}$ evaluations (about 500 generations) depending on the driving cycle and the problem formulation.

\section{Optimal sizing results}

An example of the derived method is proposed on a compact-class vehicle with the characteristics showed in Tab. 8 and in $\S 2$. For this study, we consider urban, road and highway driving cycles [30].

\subsection{Global analysis for three driving cycles}

The Pareto fronts for the three driving cycles are presented in Fig. 5. For a given driving cycle, all the evaluated sizings are represented considering two objectives: fuel consumption and battery number.

The absolute improvement of the fuel consumption is about $10 \%$ in comparison to the initial design (see $\S 2$ ) for the three driving cycles and for $N_{b a t}=28$. For all the driving cycles and the two formulations, one can see the improvement of the fuel consumption while the battery number increases. This phenomenon is mainly explained by the regenerative braking and the decreasing of the battery losses. Afterwards, the fuel consumption is no longer improved because of the increasing weight of the vehicle.

Comparing the two formulations, we observe that the second formulation, taking into account 15 unknown variables, always gives better results, which is due to the fact that the geometrical variables are uncorrelated. Between the two formulations, we notice a maximal difference of 1.8, 1.7 and $0.8 \%$ for the optimal fuel consumption for the urban, road and highway driving cycles. As a consequence, an optimization process is approximately three times longer for the formulation SP2. For instance, the SP1 for the urban case takes about $4 h$ to converge (with a i7-2630QM 2.0 GHz processor, 8Go RAM and Windows 7 Pro) whereas the SP2 takes about $12 h$.

\subsection{Optimal sizing analysis for the urban driving cycle}

Optimal sizings for the two formulations are described in Tab. 5 for the urban driving cycle. For the two formulations, we observe a coherent increasing of the EM standard power with respect to the number of battery cells. In parallel, the decreasing of the ICE 


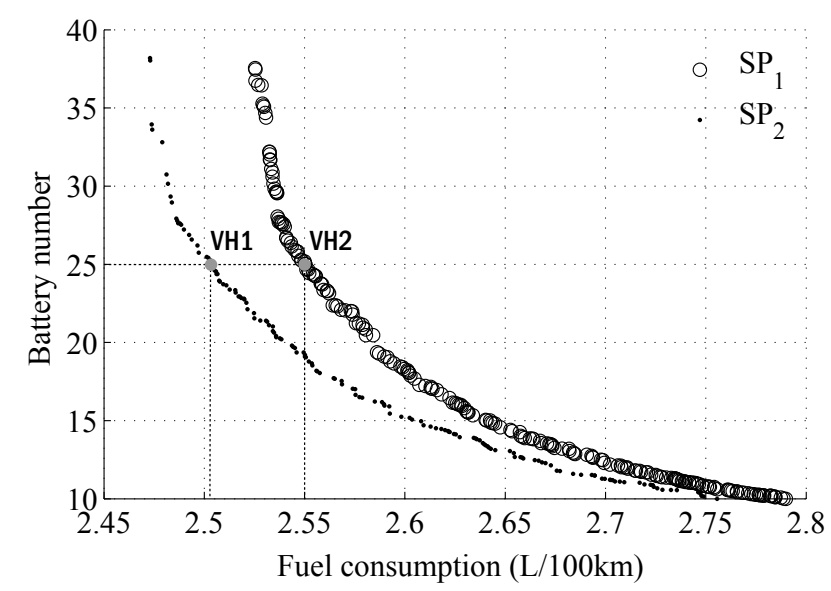

(a) Case Urban

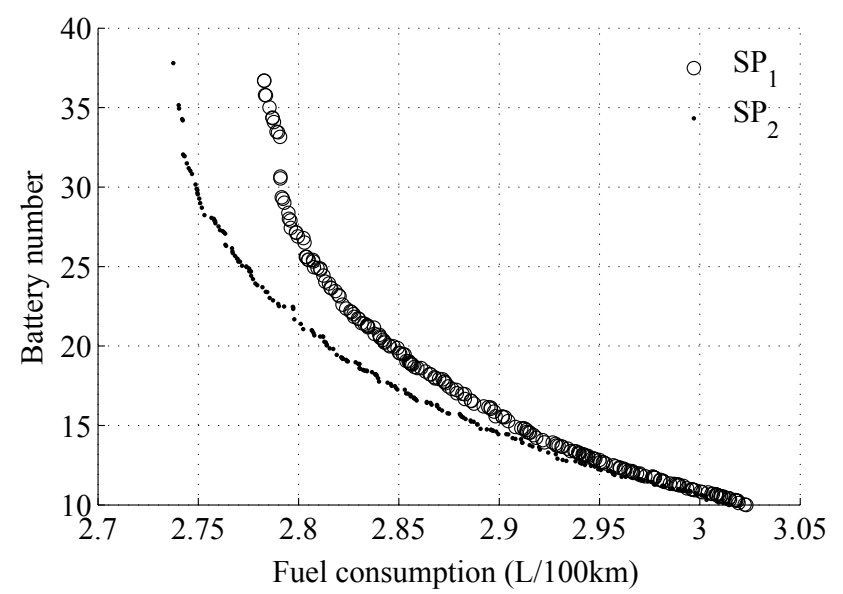

(b) Case Road

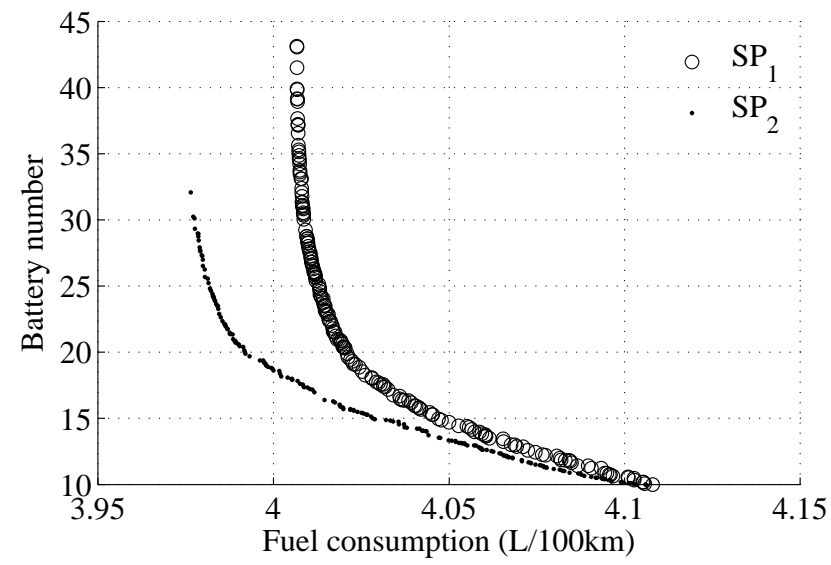

(c) Case Road

Figure 5: Pareto fronts for three driving cycles - comparison between SP1 and SP2

standard power allows a decrease of the fuel consumption while respecting the dynamic performances.

Comparing the optimal results of the two formulations, we notice small differences for the optimal ratios $k_{c p l}$ and $k_{r e d}$ that influence the operating points of the EM. For display reasons, geometrical variables of the EM are not given in Tab. 5 . 


\begin{tabular}{ccccccc|c} 
& $N_{\text {bat }}$ & $\begin{array}{c}P_{\text {bat }} \\
(k W)\end{array}$ & $\begin{array}{c}P_{m e} \\
(\mathrm{~kW})\end{array}$ & $\begin{array}{c}P_{\text {ice }} \\
(\mathrm{kW})\end{array}$ & $k_{\text {cpl }}$ & $k_{\text {red }}$ & $\begin{array}{c}J_{1} \\
(l / 100 \mathrm{~km})\end{array}$ \\
\cline { 2 - 7 } VH1 & 10 & 10,5 & 12,5 & 46,9 & 1,050 & 2,663 & 2,790 \\
& 25 & 26,2 & 15,4 & 32,1 & 0,9092 & 2,754 & 2,552 \\
& 35 & 36,8 & 19,5 & 27,6 & 0,7930 & 3,108 & 2,530
\end{tabular}

(a) SP1

\begin{tabular}{cccccccc|c} 
& $N_{\text {bat }}$ & $\begin{array}{c}P_{\text {bat }} \\
(k W)\end{array}$ & $\begin{array}{c}U_{\text {bus }} \\
(V)\end{array}$ & $\begin{array}{c}P_{\text {me }} \\
(k W)\end{array}$ & $\begin{array}{c}P_{\text {ice }} \\
(k W)\end{array}$ & $k_{\text {cpl }}$ & $k_{\text {red }}$ & $\begin{array}{c}J_{1} \\
(l / 100 k m)\end{array}$ \\
\cline { 2 - 8 } VH2 & 10 & 10,5 & 201 & 18,62 & 47,40 & 0,7637 & 2,644 & 2,756 \\
& 25 & 26,2 & 221 & 29,88 & 31,92 & 0,5956 & 2,658 & 2,506 \\
& 35 & 36,8 & 233 & 31,79 & 28,04 & 0,5999 & 2,794 & 2,479
\end{tabular}

(b) SP2

Table 5: Optimal sizing results for SP1 and SP2 for the urban driving cycle

\subsection{Comparative analysis for the urban case and $N_{b a t}=25$}

The optimal shapes of the EM are represented in Fig. 6 for $N_{b a t}=25$. In the rest of the paper, the optimal sizings for the urban driving cycle and $N_{b a t}=25$ are noted VH1 and VH2.

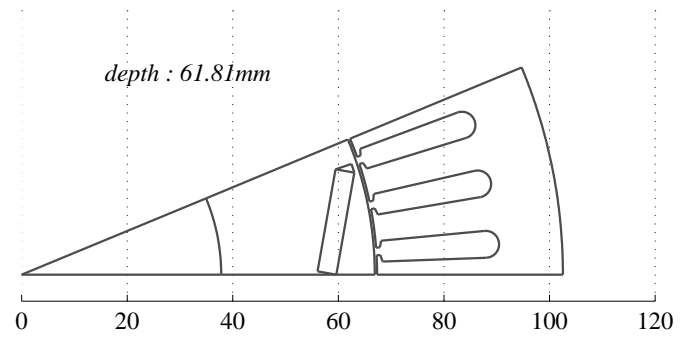

(a) VH1

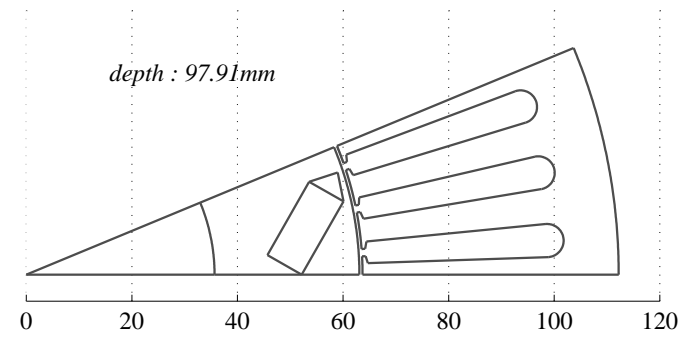

(b) VH2

Figure 6: Geometrical representations of optimal EM for the urban driving cycle and $N_{\text {bat }}=25$

The optimal EM resulting from the second formulation gets bigger dimensions (external radius and depth) and leads to a powerful electrical machine. In addition, we notice a big difference in the use of raw material (copper and magnet) between the two formulations. Volumes of raw materials are presented in Tab. 6 for VH1 and VH2. The increasing volume of magnets tends to increase the electromotive force and the synchronous torque. In addition, the salience of the EM is bigger and tends to increase the reluctance torque. The section of the copper allows to reduce Joule losses in the windings.

Efficiency maps of sizings VH1 and VH2 and operating points over the urban driving cycle are presented in Fig. 7. Firstly, we note that both EM are over-sized considering the 


\begin{tabular}{ccc} 
& $\begin{array}{c}\text { magnet volume } \\
\left(\times 10^{-4} \mathrm{~m}^{3}\right)\end{array}$ & $\begin{array}{c}\text { copper volume } \\
\left(\times 10^{-3} \mathrm{~m}^{3}\right)\end{array}$ \\
\hline $\mathcal{V H} \mathcal{H}_{1}$ & 0,961 & 0,77 \\
$\mathcal{V H} \mathcal{H}_{2}$ & $1,87(+95,1 \%)$ & $1,44(+86,3 \%)$
\end{tabular}

Table 6: Raw material volumes

operating points. This is due to a compromise between the standard power of the ICE because the total installed power is mainly constrained to the dynamical performances.

One can see the differences on the torque limits. It is mainly explained by the copper section difference and the maximal voltage available (see Fig. 6). For both efficiency maps, the average operating speeds are adapted to the maximum efficiency by the coupling ratios $k_{c p l}$ and $k_{r e d}$.

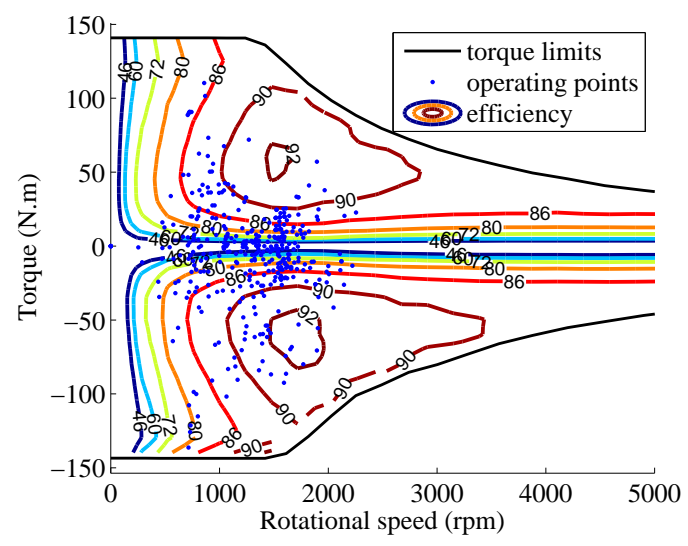

(a) VH1

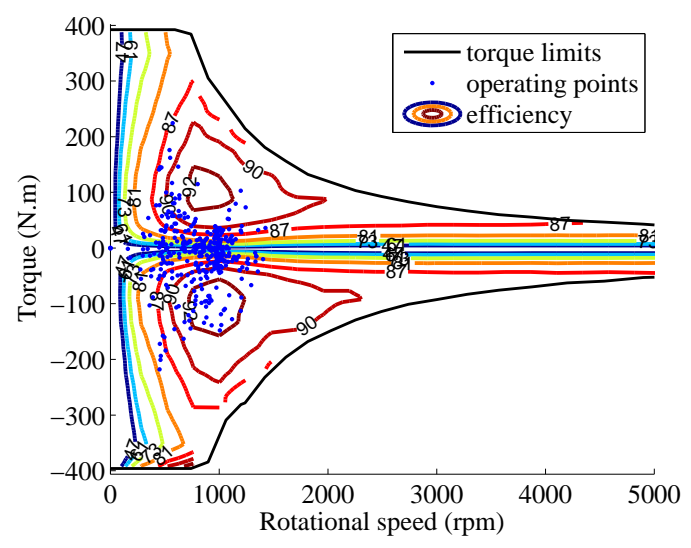

(b) $\mathrm{VH} 2$

Figure 7: Efficiency maps and operating points of VH1 and VH2 on the urban driving cycle

\begin{tabular}{ccccc} 
& $\begin{array}{c}J_{1} \\
(l / 100 \mathrm{~km})\end{array}$ & $\begin{array}{c}S F C_{m} \\
(\mathrm{~g} /(\mathrm{kW} . h))\end{array}$ & $\begin{array}{c}\eta_{m e} \\
(\%)\end{array}$ & $\begin{array}{c}\text { Energy } \\
\left(W . h . \mathrm{km}^{-1}\right)\end{array}$ \\
\hline $\mathcal{V H}_{1}$ & 2,552 & 216,9 & 83,90 & 96,21 \\
$\mathcal{V H}_{2}$ & 2,506 & 217,3 & 87,33 & 94,79
\end{tabular}

Table 7: Efficiency analysis for the urban driving cycle

The mean specific fuel consumption $\left(S F C_{m}\right)$ and the mean efficiency of the EM $\left(\eta_{m e}\right)$ are presented in Tab. 7. One can see an improvement of $3.34 \%$ of the EM efficiency over the whole cycle. Over the urban driving cycle, the fuel consumption gain between VH1 and VH2 is mainly explained by a better use of the EM. 


\section{Conclusion}

In the paper, the optimization of the electrical machine of a hybrid vehicle was discussed. First, a parametrized model of the EM has been developed and implemented in the model of a parallel-HEV. A particular attention was paid to the magnetic circuit model of the EM. Then, the model of the parallel-HEV was integrated in a global joint optimization of the sizing of the parallel-HEV and the energy management. This optimization process is a multi-objective optimization taking into account the optimal energy management, the main sizing variables of the drive train and the geometry of the electrical machine.

In a previous work, the comparison between the MCM and the MSFM emphasized the importance of an accurate model in an optimization context [10]. The MSFM tends to be optimistic in case of downsizing of the EM. The benefit of the magnetic circuit model is to take into account the geometrical characteristics of the EM. Thus, MCM, or fine models, appear to be a must, in order to size the electrical machine in a hybrid application.

In this paper, two formulations of the sizing problem have been developed and involved in the optimization process for three different driving cycles. The first formulation considers a scaling factor on the geometrical parameters, thus, it leads to a smaller search space. The second formulation, considering the geometrical parameters to be uncorrelated, leads to better results in terms of fuel consumption (about 1\%). Moreover, as the Pareto front is different, this method can lead to a different choice of the standard power of the EM and the final size of the battery pack. This way, we designed the EM for the hybrid application. Nevertheless, this formulation considers numerous optimization variables and takes about 15 hours to converge, compared to about 4 hours with a scaling factor method. In addition, it leads to an important use of raw materials. This study assumes only two cost functions, the fuel consumption and the battery number. For a simple model of the EM, it can be sufficient, but for more complex model (i.e. the formulation taking into account all the geometrical parameters of the EM), a compromise between the use of raw materials and the fuel consumption could be considered. Further work will also include a robustness study regarding the driving cycles.

\section{Appendix}

The initial characteristics of the initial hybrid vehicle and the electrical machine are shown in Table 8 . 


\begin{tabular}{|c|c|c|c|}
\hline \multicolumn{2}{|c|}{ Notation Name } & VALUE & UNIT \\
\hline \multicolumn{4}{|c|}{ ME } \\
\hline & $\begin{array}{l}\text { vehicle total } \\
\text { weigth }\end{array}$ & 1120 & $k g$ \\
\hline \multicolumn{4}{|c|}{ ME } \\
\hline$P_{b}$ & standard power & 56.4 & $k W$ \\
\hline$\Omega_{b}$ & standard speed & 1242 & tr.min ${ }^{-1}$ \\
\hline$\Gamma_{b}$ & max. torque & 454.5 & N.m \\
\hline$U_{d c}$ & $\begin{array}{l}\text { continuous } \\
\text { voltage }\end{array}$ & 500 & $V$ \\
\hline$j_{\max }$ & $\begin{array}{lr}\max . & R M S \\
\text { current density }\end{array}$ & 12 & A. $m m^{-2}$ \\
\hline$V_{\max }$ & $\begin{array}{l}\max . \quad \text { peak } \\
\text { voltage }\end{array}$ & 295 & $V$ \\
\hline \multicolumn{4}{|c|}{ ICE } \\
\hline & type & diesel & \\
\hline$P_{\text {ice }}$ & $\max$. power & 50.6 & $k W$ \\
\hline$V_{i c e}$ & displacement & 1598 & $\mathrm{~cm}^{3}$ \\
\hline \multicolumn{4}{|c|}{ NiMh Battery pack } \\
\hline$N_{b a t}$ & $\begin{array}{l}\text { NiMh module } \\
\text { number in se- } \\
\text { ries }\end{array}$ & 28 & s.u \\
\hline$P_{\text {bat }}$ & standard power & 29.5 & $k W$ \\
\hline \multicolumn{4}{|c|}{ Gears } \\
\hline$k_{c p l}$ & coupling ratio & 2.0 & s.u \\
\hline$k_{\text {red }}$ & $\begin{array}{l}\text { reduction } \\
\text { ration }\end{array}$ & 3.2941 & s.u \\
\hline$k_{g b}$ & gearbox ratios & $\begin{array}{l}\{\emptyset, \\
41 / 11, \\
43 / 21, \\
37 / 28, \\
34 / 35, \\
31 / 41\}\end{array}$ & s.u \\
\hline
\end{tabular}

Table 8: Parallel HEV characteristics

\section{References}

[1] A. Sciarretta and L. Guzzella, "Control of hybrid electric vehicles," Control systems, IEEE, vol. 27, no. 2, pp. 60-70, 2007.

[2] V. Reinbold, L. Gerbaud, and E. Vinot, "Joint optimization of control and sizing of the parallel hev using sqp algorithm," in 13th International Workshop on Optimiza- 
tion and Inverse Problems in Electromagnetism (OIPE), (Delft), September 2014.

[3] M.-J. Kim and H. Peng, "Power management and design optimization of fuel cell/battery hybrid vehicles," Journal of Power Sources, vol. 165, no. 2, pp. 819$832,2007$.

[4] E. Vinot, R. Trigui, Y. Cheng, C. Espanet, A. Bouscayrol, and V. Reinbold, "Improvement of an evt-based hev using dynamic programming," IEEE transactions on vehicular technology, vol. 63, no. 1, pp. 40-50, 2014.

[5] O. Sundström, L. Guzzella, and P. Soltic, "Optimal hybridization in two parallel hybrid electric vehicles using dynamic programming," in Proceedings of the $1^{\text {th }}$ IFAC world congress, pp. 4642-4647, 2008.

[6] C. Desai and S. S. Williamson, "Optimal design of a parallel hybrid electric vehicle using multi-objective genetic algorithms," in Vehicle Power and Propulsion Conference, (VPPC), pp. 871-876, IEEE, 2009.

[7] S. Buerger, B. Lohmann, M. Merz, B. Vogel-Heuser, and M. Hallmannsegger, "Multiobjective optimization of hybrid electric vehicles considering fuel consumption and dynamic performance," in Vehicle Power and Propulsion Conference (VPPC), 2010 IEEE, pp. 1-6, IEEE, 2010.

[8] C. Bertram, D. Buecherl, A. Thanheiser, and H. Herzog, "Multi-objective optimization of a parallel hybrid electric drive train," in Vehicle Power and Propulsion Conference (VPPC), 2011 IEEE, pp. 1-5, IEEE, 2011.

[9] A. Kimura, T. Abe, and S. Sasaki, "Drive force control of a parallel-series hybrid system," JSAE review, vol. 20, no. 3, pp. 337-341, 1999.

[10] V. Reinbold, L. Garbuio, E. Vinot, and L. Gerbaud, "Magnetic circuit model: a quick and accurate sizing model for electrical machine optimization in hybrid vehicles," in Vehicle Power and Propulsion Conference, (Coimbra, Portugal), September 2014.

[11] T. Hofman, M. Steinbuch, R. Van Druten, and A. Serrarens, "Rule-based energy management strategies for hybrid vehicles," International Journal of Electric and Hybrid Vehicles, vol. 1, no. 1, pp. 71-94, 2007. 
[12] K. Chau, M. Cheng, and C. Chan, "Nonlinear magnetic circuit analysis for a novel stator doubly fed doubly salient machine," Magnetics, IEEE Transactions on, vol. 38, no. 5, pp. 2382-2384, 2002.

[13] M. Zeraoulia, M. E. H. Benbouzid, and D. Diallo, "Electric motor drive selection issues for hev propulsion systems: A comparative study," Vehicular Technology, IEEE Transactions on, vol. 55, no. 6, pp. 1756-1764, 2006.

[14] T. Finken, M. Felden, and K. Hameyer, "Comparison and design of different electrical machine types regarding their applicability in hybrid electrical vehicles," in Electrical Machines, 2008. ICEM 2008. 18th International Conference on, pp. 1-5, IEEE, 2008.

[15] J. Hsu, C. Ayers, and C. Coomer, "Report on toyota/prius motor design and manufacturing assessment," Federal Register, vol. 2011, pp. 2012-2013, 2010.

[16] Y. Cheng, R. Trigui, C. Espanet, A. Bouscayrol, and S. Cui, "Specifications and design of a pm electric variable transmission for toyota prius ii," Vehicular Technology, IEEE Transactions on, vol. 60, no. 9, pp. 4106-4114, 2011.

[17] J. Hsu, C. Ayers, C. Coomer, R. Wiles, S. Campbell, K. Lowe, and R. Michelhaugh, Report on Toyota/Prius Motor Torque-Capability, Torque-Property, No-Load Back EMF, and Mechanical Losses. United States. Department of Energy, 2004.

[18] D. Buecherl, C. Bertram, A. Thanheiser, and H. Herzog, "Scalability as a degree of freedom in electric drive train simulation," in Vehicle Power and Propulsion Conference (VPPC), pp. 1-5, IEEE, 2010.

[19] B. Du Peloux, L. Gerbaud, F. Wurtz, V. Leconte, and F. Dorschner, "Automatic generation of sizing static models based on reluctance networks for the optimization of electromagnetic devices," IEEE Transactions on Magnetics, vol. 42, no. 4, pp. 715$718,2006$.

[20] H. Dogan, F. Wurtz, A. Foggia, and L. Garbuio, "Analysis of slot-pole combination of fractional-slots pmsm for embedded applications," in Electrical Machines and Power Electronics and 2011 Electromotion Joint Conference (ACEMP), 2011 International Aegean Conference on, pp. 611-615, IEEE, 2011.

[21] G. Bertotti, "General properties of power losses in soft ferromagnetic materials," IEEE Transactions on Magnetics, vol. 24, no. 1, pp. 621-630, 1988. 
[22] F. Deng, "An improved iron loss estimation for permanent magnet brushless machines," IEEE Transactions on Energy Conversion, vol. 14, no. 4, pp. 1391-1395, 1997.

[23] G. Feix, S. Dieckerhoff, J. Allmeling, and J. Schonberger, "Simple methods to calculate igbt and diode conduction and switching losses," in 2009 13th European Conference on Power Electronics and Applications, 2009.

[24] F. Magnussen, Y.-K. Chin, J. Soulard, A. Broddefalk, S. Eriksson, and C. Sadarangani, "Iron losses in salient permanent magnet machines at field-weakening operation," in Industry Applications Conference, $39^{\text {th }}$ IAS Annual Meeting, vol. 1, IEEE, 2004.

[25] E. Silvas, T. Hofman, and M. Steinbuch, "Review of optimal design strategies for hybrid electric vehicles," IFAC Workshop on Engine and Powertrain Control, Simulation and Modeling, vol. 3, no. 1, pp. 57-74, 2012.

[26] E. Vinot, R. Trigui, B. Jeanneret, J. Scordia, and F. Badin, "Hevs comparison and components sizing using dynamic programming," in Vehicle Power and Propulsion Conference (VPPC), pp. 314-321, IEEE, 2007.

[27] D. E. Kirk, Optimal control theory: an introduction. Courier Dover Publications, 2012 .

[28] K. B. Wipke, M. R. Cuddy, and S. D. Burch, "Advisor 2.1: a user-friendly advanced powertrain simulation using a combined backward/forward approach," IEEE Transactions on Vehicular Technology, vol. 48, no. 6, pp. 1751-1761, 1999.

[29] K. Deb, A. Pratap, S. Agarwal, and T. Meyarivan, "A fast and elitist multiobjective genetic algorithm: Nsga-ii," IEEE Transactions on Evolutionary Computation, vol. 6, no. 2, pp. 182-197, 2002.

[30] M. Andr, "Driving pattern analysis and driving cycles - European Development of Hybrid Technology approaching Zero Emission Mobility (HYZEM)," Tech. Rep. 9709, INRETS LEN, 1997. 